\title{
Plasma Therapy in the fight against Sars-CoV-2: what boils in the pot.
}

\author{
Antonio Vitiello ${ }^{1}$, Francesco Ferrara ${ }^{1}$, Raffaele La Porta ${ }^{2}$, and Chiara Pelliccia ${ }^{3}$ \\ ${ }^{1}$ Azienda Unità Sanitaria Locale Umbria 1 \\ ${ }^{2}$ ASUR Marche \\ ${ }^{3}$ Azienda Unità Sanitaria Locale Umbria 2
}

May 11, 2020

\begin{abstract}
The global pandemic from Sars-cov-2 has down caused thousands of deaths worldwide, triggering a health crisis in the various countries involved, with few precedents in history. To date there are no vaccines for prophylaxis, and there are no antivirals directed against the virus. Among the therapeutic options that have shown effectiveness is passive immunization with immune plasma from convalescent patients cured of the infection. Plasma collected from patients cured of Sars-cov-2 infection is rich in antibodies that neutralized the pathogen. Plasma therapy has already demonstrated its efficacy in other epidemics, such as Sars-Cov and MERS. To date, there are limited data for its use in sars-Cov-2 infection, both for prophylaxis and treatment, but the few existing data bode well for the scientific world. Many questions are still unresolved, when to administer it? At what dosage? When is it most appropriate to take the plasma from the cured patient? Are there different answers depending on gender and age? Certainly in view of the high number of patients infected and cured by Sars-Cov-2, there could be a high amount of plasma from donor patients. In this article we want to give an overview on a current and important topic in the perspective of the battle against the new Sars-Cov-2, analysing the therapeutic successes in past epidemics, the clinical data currently available, the future prospect and an expert opinion.
\end{abstract}

\section{Introduction}

\section{Sars-Cov-2}

Since November 2019, cases of lethal infection associated with a new coronavirus, SARS-Cov-2 (COVID-19), have occurred in China, and then rapidly in several countries around the world.. At the time of writing this article are recorded about 3.7 million infected in the world with about 1.25 million healed and about 265 thousand deaths. This global pandemic has triggered a global health crisis with few precedents in human history. From the various studies carried out, the phase of the infection has been divided into three phases, the first with mild or absent symptoms where the immune system fights the virus, the second where the symptomatology is more serious and there is a hyperactive inflammatory system, the third where the immune system is altered and there is a generalized inflammatory state that can cause serious and fatal lung lesions and multi-organ dysfunction. (1-2) To date, there are no vaccines for the virus, nor direct antivirals. The drugs used are registered antivirals for other viral infections, or drugs that act on the generalized inflammatory state such as immunomodulants. While we are waiting for the discovery of an effective vaccine or direct antiviral against Sars-cov-2 soon, excellent hopes come from the passive immunization of the infected patient with the administration of convalescent plasma from patients already cured of the infection. The Plasma therapy (PT) has already shown benefits in the treatment of prophylaxis and treatment for Sars-Cov-2 infection.(3-4)

\section{The Plasma Therapy}


Plasma therapy (PT) is a passive immunization technique, which involves the administration of plasma collected from individuals who have recovered from an infection and developed antibodies against the pathogen, in a patient who is sick and infected with the same type of virus. PT can prevent a clinical infection, or prevent the worsening of an existing infection, by helping the host organism to fight the virus. Today, PT is mainly based on pooled immunoglobulin preparations that contain high concentrations of antibodies. However, some evidence shows that PT is more effective when administered prophylactically or used early after the onset of symptoms (5-6).

\section{Results with past epidemics}

Passive immunization techniques have been in use for about a century. With PT, other Sars-cov-2-like epidemics, such as those caused by the severe acute respiratory syndrome (SARS-CoV) coronavirus (7), for MERS-CoV (8), avian influenza $\mathrm{A}(\mathrm{H} 5 \mathrm{~N} 1)(9)$ virus and influenza $\mathrm{A}(\mathrm{H} 1 \mathrm{~N} 1)(10)$, have already had excellent results. In these epidemics similar in some clinical and pathological aspects to that of Sars-Cov-2, in patients with PT administration there was a reduction in mortality and a faster recovery.

\section{The PT mechanism of action}

Antibodies are a key component of the immune system and play an important role in the fight against infectious agents. They have the ability to bind specifically to non-self antigens (infectious microorganisms such as viruses, bacteria, toxins), perform functions such as mucosal protection, opsonizing and complement activation, are produced by B lymphocytes, transformed to perform this task, following specific stimuli, into plasma cells. Together with T lymphocyte receptors (TCR) and molecules of the major histocompatibility complex MHC are molecules capable of binding the antigen to a highly specific degree. (11-12) Antibodies present in the immune plasma, (i.e. "convalescent") in the same way, mediate their therapeutic effect through a variety of mechanisms. The antibody can bind to a certain pathogen (e.g. virus), thus directly neutralizing its infectivity, increasing phagocytosis, complement activation. (13-14). It is important to emphasize that the administration of plasma therapy with antibodies currently offers the only and accessible short-term strategy to confer immediate immunity to individuals to fight the infection, especially in individuals with weakened immune systems due to other diseases or drug therapies. Pending an effective vaccine, and an antiviral or monoclonal antibody directed against Sars-cov-2, PT anti-SARS-CoV-2 is the only therapeutic strategy that is immediately available for use to prevent and treat COVID-19.

\section{Evidence with currently epidemic Sars-cov-2}

There is some evidence demonstrating the efficacy of PT in COVID-19 patients. In a pilot study conducted in China on 10 severe COVID-19 patients, an improvement in clinical symptoms was recorded in all patients within a few days of PT administration (15); in 7 patients, transfusion of PT was associated with a decrease in viral load. Five cases of COVID-19 severe patients were described, who reported a rapid improvement in clinical status following transfusion, with convalescent plasma (IgG titers SARS-CoV-2 >1000) (16). These two studies also have a limited sample. In order to assess the effectiveness of plasma convalescence therapy, another study conducted in patients with severe COVID-19 showed that PT resulted in symptom alleviation, changes in radiological abnormalities and laboratory tests and that no obvious adverse effects were observed during treatment. (17) In addition, a review was carried out to assess the effectiveness of PT in patients with COVID-19. The results reported that PT in patients with COVID-19 can reduce mortality in critical patients, the increase in neutralizing antibody titers and the disappearance of SARS-CoV-2 RNA was observed in almost all patients after therapy, a reduction in clinical symptoms after administration of convalescent plasma. Based on the results of this review, although data are limited, PT in the COVID-19 patient appears safe, clinically effective and reduces mortality. (18)

\section{TRIALS on going}

To date, there are no results from large, well-designed multicentre clinical trials establishing the efficacy of $\mathrm{PT}$ in patients with COVID-19.During this period numerous ttrials worldwide are being conducted to assess the efficacy and safety of PT in COVID-19 patients. Six clinical trials are underway in Italy.the first. Table 
1 shows the studies in progress in Italy. In most of these studies it will use the dose based on experience published in the literature, $250-300 \mathrm{~mL}$ of convalescent plasma will be used to treat each of the patients recruited a maximum of 3 times in 5 days. The main outcomes that will be evaluated are the trend in the concentration of inflammatory markers, the viral load, the remission of clinical symptoms, the number of healings. The various trials conducted in Italy and around the world will provide important EBM that will provide valuable information on the real effectiveness of PT in COVID-19 patients, the right dose to use, the time to take plasma from cured patients, the safety of therapy. (tab.1)

\begin{tabular}{lll}
\hline & Study Reference & Conditions \\
\hline 1 & Hyperimmune Plasma for Critical & COVID-19 \\
& Patients With Covid-19 & \\
& Early transfusIon of Convalescent & Coronavirus Disease 2019 \\
& Plasma in Elderly & \\
& Covid-19 Patients. to Prevent & \\
& Disease Progression. & \\
& Clinical Performance of the & Coronavirus Infections \\
& VivaDiag Covid-19 lgM / IgG & \\
& Rapid Test in Early Detecting the & \\
& Infection of Covid-19 & \\
& Clinical and Biological Predictors & COVID-19 \\
& of Covid-19 Disease in Older & \\
& Patients & \\
& Convalescent Antibodies Infusion & Pneumonia, Ventilator-Associated \\
& in Critically Ill Covid-19 Patients & Coronavirus Infection \\
& Prognostic Factors Keeping Track & Pneumonia, Viral \\
& for Covid-19 Pneumonia & Hypertension \\
& & Diabetes Mellitus \\
\hline
\end{tabular}

Table 1 : Italian ongoing studies ( source: clinical trials.gov)

Optimum dosage

Evidence from the treatment of past epidemics with PT, and the results currently available indicate that the correct dose of convalescent plasma to be administered may be variable depending on a different indication, whether it is in prophylaxis or treatment. The reported studies have used approximately $(200 \mathrm{~mL})$ units of plasma (19), however, in the various clinical studies currently underway, therapeutic regimens for postexposure prophylaxis and therapeutic regimens for the treatment of infection have been proposed. The duration of effectiveness of PT is not known, but it is hypothesized that to last from weeks to a few months (20) . For a patient of about $80 \mathrm{Kg}$, the standard dose to be used for the treatment should be in dependence of the various supporting studies, about $250 \mathrm{ml}$ of plasma, in the pediatric population (experiments are being planned), there will be a need for doses according to body weight. However, there are currently levels of uncertainty about the optimal dose.

Potential Risks

The method of convalescent PT has been used daily in many hospitals for years. Convalescent PT against SARS-Cov-2 differs from standard plasma only in the presence of neutralizing antibodies directed against Sars-Cov-2, therefore the transfusion risks could be considered not different from those of standard plasma. An open question could be the risk of infection transmission. In COVID-19 patients this should obviously not be considered because they are already infected, but in the case of prophylactic use yes. However, these are theoretical risks, as no transmission of respiratory infection has ever been reported. viral by plasma transfusion. SARS-CoV-2 is probably not considered a relevant transfusion virus of infection.(21- 
22). However, it should be considered that donors will have to meet certain requirements, such as waiting a few weeks after the resolution of their symptomatology and above all must also be negative for SARS-CoV-2, determined by diagnostic molecular tests.

\section{Expert opinion}

Few controlled studies have been carried out to assess the efficacy of convalescent PT in viral infections, perhaps for its application only in emergencies during epidemics. Surely the many ongoing studies will give us a better picture of the situation. There are still many unresolved issues such as the right dose to be administered in the various categories of patients in both prophylaxis and treatment. In addition, we need to clarify the duration of effectiveness of PT from the moment it is administered and whether it should be used in prophylaxis in the categories of individuals most at risk of infection, such as elderly patients, immunodepressed patients, healthcare professionals, individuals with cardiovascular and respiratory diseases, residents of nursing homes. Prevention would bring direct clinical benefit to people at risk. In addition, the benefits for society as a whole would be far-reaching, including the protection of healthcare workers working at the forefront of the COVID-19 pandemic. In addition, the effectiveness of convalescent PT on healing and the effect it can have in preventing worsening clinical conditions should also be investigated. Another aspect to be clarified is the right time to take the convalescent plasma from the healed donor patient, advised that the amount of antibodies neutralizing the virus changes over time. However, it is likely that convalescent PT can be therapeutically effective in COVID-19 prophylaxis and in stages one, two and three of the infection, even in polypharmacy with antivirals.

\section{Conclusions}

Pending the development of an effective vaccine against COVID-19, and the development of direct antivirals, a therapeutic hope may arise from the use of PT taken from patients treated with COVID-19. Currently, based on the successes achieved against past epidemics, such as Sars-Cov and MERS, similar to the current Sars-Cov-2, and based on the first efficacy data from early studies, it is expected that human plasma therapy with COVID-19 cured patients will be an effective and safe therapy for both treatment and post-exposure prophylaxis. However, the importance and validity of this therapeutic treatment will be defined by the various ongoing clinical studies.

\section{MAIN STATEMENTS}

I, the undersigned, Francesco Ferrara and any other author, declare that:

- We have no conflict of interest;

- We have not received funding;

- There are no sensitive data and no patients were recruited for this study;

- The document does not conflict with ethical legislation.

Regards

The authors

\section{References}

1. P. Zhou et al., A pneumonia outbreak associated with a new coronavirus of probable bat origin. Nature 579, 270-273 (2020).

2. Chen et al., Epidemiological and clinical characteristics of 99 cases of 2019 novel coronavirus pneumonia in Wuhan, China: A descriptive study. Lancet 395, 507-513 (2020).

3. World Health Organization, Coronavirus disease (COVID-19) Pandemic. https://www. who.int/emergencies/diseases/novel-coronavirus-2019.

4. H. Lu. Drug treatment options for the 2019-new coronavirus (2019-nCoV). Biosci. Trends 14, 69

5. Casadevall A, and Pirofski LA. Antibody-mediated regulation of cellular immunity and the inflammatory response. Trends Immunol. 2003;24(9):474-8. 4. 
6. Casadevall A, and Scharff MD. Serum therapy revisited: animal models of infection and development of passive antibody therapy.Antimicrob Agents Chemother. 1994;38(8):1695-702.

7. Stockman LJ, Bellamy R, Garner P. SARS: systematic review of treatment effects. PLoS Med. 2006;3:e343. 10.1371/journal.pmed.0030343

8. Yaseen M. Arabi et al. Feasibility of Using Convalescent Plasma Immunotherapy for MERS-CoV Infection. Saudi Arabia Emerg Infect Dis. 2016 Sep; 22(9): 1554-1561. doi: 10.3201/eid2209.151164

9. Hung IF, To KK, Lee CK, Lee KL, Yan WW, Chan K, et al.Hyperimmune IV immunoglobulin treatment: a multicenter double-blind randomized controlled trial for patients with severe 2009 influenza A(H1N1) infection. Chest. 2013;144:464-73. 10.1378/chest.12-2907

10. Hung IF, To KK, Lee CK, Lee KL, Chan K, Yan WW, et al.Convalescent plasma treatment reduced mortality in patients with severe pandemic influenza A (H1N1) 2009 virus infection. Clin Infect Dis. 2011;52:447-56. 10.1093/cid/ciq106

11. Van Erp EA, Luytjes W, Ferwerda G, and van Kasteren PB.Fc-Mediated Antibody Effector Functions During Respiratory Syncytial Virus Infection and Disease. Front Immunol. 2019;10:548.

12. Gunn BM, Yu WH, Karim MM, Brannan JM, Herbert AS, Wec AZ, et al.A Role for Fc Function in Therapeutic Monoclonal Antibody-Mediated Protection against Ebola Virus. Cell Host Microbe. 2018;24(2):221-33.e5. 15.

13. Zhang JS, Chen JT, Liu YX, Zhang ZS, Gao H, Liu Y, et al. A serological survey on neutralizing antibody titer of SARS convalescent sera. J Med Virol. 2005;77(2):147-50. 16.

14. Cheng Y, Wong R, Soo YO, Wong WS, Lee CK, Ng MH, et al. Use of convalescent plasma therapy in SARS patients in Hong Kong. Eur J Clin Microbiol Infect Dis. 2005;24(1):44-6.

15. Duan K, Liu B, Li C, Zhang H, Yu T, Qu J, et al. The feasibility of convalescent plasma therapy in severe COVID-19 patients: a pilot study. medRxiv. 2020:2020.03.16.20036145. 23

16. Shen C, Wang Z, Zhao F, Yang Y, Li J, Yuan J, et al. Treatment of 5 Critically Ill Patients With COVID-19 With Convalescent Plasma.JAMA. 2020. 22.

17. Ye $\mathrm{M}$ et al. Treatment with convalescent plasma for COVID-19 patients in Wuhan, China.J Med Virol. 2020 Apr 15. doi: 10.1002/jmv.25882.

18. Rajendran K et al. Convalescent plasma transfusion for the treatment of COVID-19: Systematic review.

19. Duan K, Liu B, Li C, Zhang H, Yu T, Qu J, et al. The feasibility of convalescent plasma therapy in severe COVID-19 patients: a pilot study. medRxiv. 2020:2020.03.16.20036145.

20. Casadevall A. Passive antibody administration (immediate immunity) as a specific defense against biological weapons. Emerg Infect Dis. 2002;8(8):833-41.

21. FDA. Electronic Code of Federal Regulations: 630.30 Donation suitability requirements. https://www.accessdata.fda.gov/scripts/cdrh/cfdocs/cfcfr/CFRSearch.cfm?fr=630.3. Updated March 17, 2020 Accessed March 19, 2020

22. Wang W, Xu Y, Gao R, Lu R, Han K, Wu G, et al. Detection of SARS-CoV-2 in Different Types of Clinical Specimens. JAMA. 2020. 